\title{
Satisfiability Transition and Experiments on a Random Constraint Satisfaction Problem Model
}

\author{
Wei $\mathrm{Xu}^{1}$ \\ ${ }^{1}$ School of Automation and Electrical Engineering, University of Science and \\ Technology Beijing, Beijing 100083, China \\ ${ }^{1}$ xuwei_225@163.com
}

\begin{abstract}
Random constraint satisfaction problem models play a significant role in computer science. They provide instances for benchmarking algorithms, help inform the design of algorithms and heuristics, and provide insight into problem nardness. In this paper, model $R A$ which was revised from classical model A, was stlidied. When parameter $k$ satisfied $k \geq 3$, model $R A$ has the same satisfiability transition with model $\hat{R} B$, which was revised from classical model $B$ and has already got considerable theoretical and practical studies. We also compared the performances of fundamental algorithms on model RA with on model RB.
\end{abstract}

Keywords: Constraint satisfaction problem, Model RB, Satisfiability transition

\section{Introduction}

Constraint programming [1] is a general framework for describing and modeling realworld constraint problems and solving them, it is wildly studied. It is successfully applied to many domains, such as scheduling, planning, vehicle routing, configuration, circuit design, and bioinformatics. Constraint satisfaction problem (CSP) is an important part of constraint programming. CSP arises in a large spectrum of scientific disciplines, such as computer science, information theory, and statistical physics.

A typical CSP instance involves a large set of discrete variables, each one taking a finite number of values, and a collection of constraints, each one involving a few variables and forbidding some of their joint values. A solution is an assignment, or joint values of all variables, that satisfies 11 the constraints simultaneously.

For some purposes, we may wish to generate CSP instances on demand. There should be a model to generate random CSP instances automatically, then experiments were realized and algorithms were-tested. A lot of benchmarks based on different CSP models are working on area of algorthm competitions. With the experiments and analyses of random models, better strateges and heuristics would be proposed. Another reason to research on CSP models is understanding computational difficulty. The structure of solution space may be one of the links $\mathrm{n}$ resolving the $\mathrm{P}=\mathrm{NP}$ question. Classical random models were proposed $[2,3]$, denoted by A, B, C and D. After that, many alternatives appeared [4-8].

Structure of solution space is thought to have critical effects on problem hardness and algorithm ability. Satisfiability transition was first found in experiment [9]. From then on, a lot of works in computer science were focused on a proof of transition phenomenon. Friedgut, et al. [10] made a big step, they proved for k-SAT that satisfiability has sharp threshold. Achlioptas, et al. [11] proved that clustering transition exists for some models. Physicists applied cavity method on CSPs to calculate the transition points $[12,13]$. There are very few 
CSP models which can be proved to have a satisfiability transition in rigorous ways, model $\mathrm{RB}$ is one of them.

Model RB, revised from classical model B, is a type of CSPs model with growing domains. It was proposed by $\mathrm{Xu}$ and $\mathrm{Li}$ in reference [5]. In the same paper they proved that the model have exact satisfiability transition. The forced satisfiable instances generated from Model RB are hard to solve [14, 15], and they are widely used in various kinds of algorithm competitions. Model RB has got considerable attentions [16-21].

This paper is organized as follows. We will firstly give the definitions of revised models, and explain the major difference between classical and revised models. In section 3, we will focus on satisfiability transition of model RA. In section 4, we will do experiments on RA and $\mathrm{RB}$, and show RA and RB in some sense are similar.

\section{Revised models}

Both classical models and revised models were introduced in [16]. Here we give the definitions of model RB and model RA. Let $k \geq 2$ be an integer let $r, \alpha>0,0<p<1$ be real numbers, let $\vec{\sigma}=\left\{\sigma_{1}, \sigma_{2}, \cdots, \sigma_{N}\right\}$ be variables, each variable takes value from domain $D=\left\{1,2, \cdots, N^{\alpha}\right\}$. A constraint involves $k$ variables, and a incompatible-set, which is a subset of Cartesian product $D^{k}$. Elements in incompatible-set are called incompatible joint values. Model $\mathrm{RB}(N, k, r, \alpha, p)$ is a probability space, we define it by giving the way to generate its instances. The steps are:

1. We select with repetition $r N \ln N$ random constraints Each random constraint is formed by selecting without repetition $k$ of $N$ variables.

2. For each constraint, we form anincompatible-set by uniformly select without repetition $p N^{\alpha k}$ elements of $D^{k}$.

Similarly, steps to generate instances of model $\operatorname{RA}(N, k, r, \alpha, p)$ are:

1'. A constraint involves $k$ vartables. There are $C_{N}^{k}$ feasible constraints, each one shows up with probability $\frac{r N \ln N}{C_{N}^{k}}$

2'. For every constraint, there are $N^{\alpha k}$ feasible joint values, each one shows up as incompatible joint alue with probability $p$.

A solution is an assignment, which satisfies all constraints. It is to say joint values in this solution do not belong to any incompatible-sets. The set of all solutions, denoted by $\mathrm{S}$, is a subset of $D^{N}$

Let $X$ be the number of solutions, $X=|\mathrm{S}|$. It is easy to see that in model RB the expectation of $X$ is $\mathrm{E}(X)=N^{\alpha N}(1-p)^{r N \ln N}$. The following lemma shows that Model RB has exact satisfiability transition, referring to $[5,19]$.

Lemma 1. Let $r_{c r}=-\frac{\alpha}{\ln (1-p)}$. If $\alpha>\frac{1}{k}, 0<p<1$ are two constants and $k, p$ satisfy the inequality $k \geq \frac{1}{1-p}$, then 


$$
\begin{aligned}
& \lim _{n \rightarrow \infty} \operatorname{Pr}(X>0)=1 \text { when } r<r_{c r} \\
& \lim _{n \rightarrow \infty} \operatorname{Pr}(X>0)=0 \text { when } r>r_{c r} .
\end{aligned}
$$

In classical and revised models, all variables have the same domains. If we draw a variable-domain plane, classical models are along horizontal line. With $N$ growing, size of domains is fixed. Revised models are along curves. With $N$ growing, sizes of domains are growing. This is the major difference of classical models and revised models. See Figure 1, revised model with different parameters are along different curves.

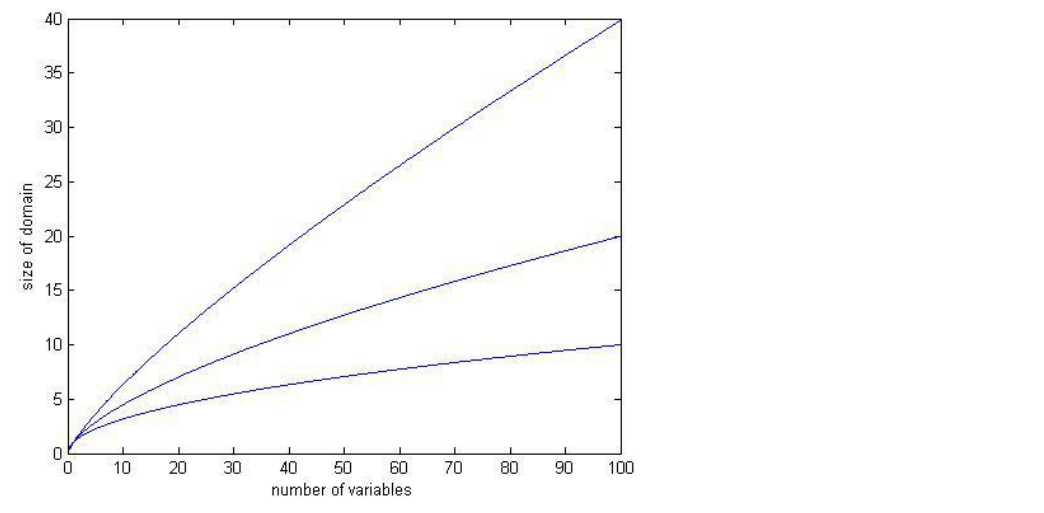

Figure 1. Revised models are along curves in variable-domain plane, instead of horizontal lines. From bottom to top three curves relate to parameters $\alpha=0.5, \alpha=0.65, \alpha=0.8$ respectively

\section{Satisfiability Transition on model RA}

We define a new model, called model RB*, the difference between model RB* and model $\mathrm{RB}$ is the first step to generate an instance. In the first step of RB*, select without repetition $r N \ln N$ random constraints, each andom constraint is formed by selecting without repetition $k$ of $N$ variables. As we cansee, every instance of model RB* is an instance of model RB.

Lemma 2. When $k \geq 3$, if we generate an $\mathrm{RB}$ instance, the probability that it is also an RB* instance goes to as $N$ growing.

Proof; Number of instances in model RB* is $P_{C_{N}^{k}}^{r N \ln N}$, number of instances in model RB is $\left(C_{N}^{k}\right)^{r N \ln N}$ where $P, C$ are symbols standing for permutation and combination. Let $n=C_{N}^{k}$, $t=r N \ln N$ then

$$
\frac{P_{C_{N}^{k}}^{r \ln N}}{\left(C_{N}^{k}\right)^{r N \ln N}}=\left(1-\frac{1}{n}\right)\left(1-\frac{2}{n}\right) \ldots\left(1-\frac{t-1}{n}\right) .
$$

When $k \geq 3, t=o\left(\frac{n}{t}\right)$, so

$$
\left(1-\frac{1}{n}\right)\left(1-\frac{2}{n}\right) \cdots\left(1-\frac{t-1}{n}\right) \geq\left(1-\frac{t}{n}\right)^{t} \rightarrow 1
$$


Lemma 3. Given constants $0<r_{0}<r_{1}, 0<p_{0}<p_{1}<1$, let $P_{1}$ be the probability that an instance of model RA $\left(r=r_{1}, p=p_{1}\right)$ is satisfiable, $P_{2}$ be the probability that an instance of model RB* $\left(r=r_{0}, p=p_{0}\right)$ is satisfiable. Then for any $\varepsilon>0$, there exists $N_{0}$, when $N>N_{0}$, $P_{1}-P_{2}<\varepsilon$.

Proof: We need to find a set of instances of model RA $\left(r=r_{1}, p=p_{1}\right)$, we use A to denote this set. Let $P_{3}$ be the probability that instance in set A is satisfiable. We need A satisfy condition 1: the probability that a RA instance belongs to set A goes to 1 with $N$ growing, and condition 2: $P_{3} \leq P_{2}$. If we have found such a set, when $N$ is big enough, the probability that a RA instance belongs to set A was bigger than $1-\varepsilon_{1}$, where $\varepsilon_{1}$ satisfied $\varepsilon_{1}<\frac{\varepsilon}{1-P+\varepsilon}$, then $P_{3}>\frac{P_{1}-\varepsilon_{1}}{1-\varepsilon_{1}}>P_{1}-\varepsilon$, so $P_{2} \geq P_{3}>P_{1}-\varepsilon, P_{1}-P_{2}<\varepsilon$. In the rest of this progf, we will find the set $\mathrm{A}$, and verify that it satisfies condition 1 and condition 2 .

We define set A: it is a set of instances of model RA $\left(r=r_{1}, p=p_{1}\right)$, every instance in set A contains more than $r_{0} N \ln N$ constraints, which contain more than $p_{0} N^{\alpha k}$ incompatible joint values.

To verify condition 1 :

For instances of RA $\left(r=r_{1}, p=p_{1}\right)$ be the number of its constraints, $X_{N}$ follows binomial distribution with Bernoulf trials $C_{N}^{k}$ success probability $\frac{r N \ln N}{C_{N}^{k}}$, expected value $C_{N}^{k} \frac{r N \ln N}{C_{N}^{k}}$, and variance $\frac{r N \ln N}{C_{N}^{k}}\left(1-\frac{N \ln N}{C_{N}^{k}}\right)$. By Chebyshev's inequality we have

$$
\begin{aligned}
& P\left(X_{N}>\frac{r_{0}+r_{1}}{2} N \ln N\right)>P\left(\left|X_{N}-r_{1} N \ln N\right|<\frac{r_{1}-r_{0}}{2} N \ln N\right) \\
& =1-P\left(\mid X_{N}\left(Y_{1} N \ln N \mid \geq \frac{r_{1}-r_{0}}{2} N \ln N\right)\right) \geq 1-\frac{C_{N}^{k} \frac{r N \ln N}{C_{N}^{k}}\left(1-\frac{r N \ln N}{C_{N}^{k}}\right)}{\left(\frac{r_{1}-r_{0}}{2} N \ln N\right)^{2}} \rightarrow 1 .
\end{aligned}
$$

That is to say almost every instance of RA $\left(r=r_{1}, p=p_{1}\right)$ have more than $\frac{r_{0}+r_{1}}{2} N \ln N$ constraints, when $N$ large.

Consider one single constraint, let $Y_{N}$ be the number of its incompatible joint values,

$$
P\left(Y_{N}>p_{0} N^{\alpha k}\right)>1-P\left(\left|Y_{N}-p_{1} N^{\alpha k}\right| \geq\left(p_{1}-p_{0}\right) N^{\alpha k}\right) \geq 1-\frac{N^{\alpha k} p_{1}\left(1-p_{1}\right)}{\left(\left(p_{1}-p_{0}\right) N^{\alpha k}\right)^{2}} \rightarrow 1 .
$$

Let $Z_{N}$ be the number of constraints which have more than $p_{0} N^{\alpha k}$ incompatible joint values, so the probability 


$$
P\left(Z_{N}>r_{0} N \ln N\right)=\sum_{i=0}^{C_{N}^{k}} P\left(Z_{N}>r_{0} N \ln N \mid X_{N}=i\right) P\left(X_{N}=i\right) .
$$

Every constraint in model RA select its incompatible joint values independently, and in each constraint the probability that incompatible joint values is more than $p_{0} N^{\alpha k}$ is $P\left(Y_{N}>p_{0} N^{\alpha k}\right)$. When $X_{N}=i, Z_{N}$ follows binomial distribution with Bernoulli trials $i$, and success probability $P\left(Y_{N}>p_{0} N^{\alpha k}\right)$. When $i>\frac{r_{0}+r_{1}}{2} N \ln N$,

$$
P\left(Z_{N}>r_{0} N \ln N \mid X_{N}=i\right) \geq P\left(Z_{N}>r_{0} N \ln N \mid X_{N}=\frac{r_{0}+r_{1}}{2} N \ln N\right) .
$$

We have

$$
\text { (1) }>P\left(Z_{N}>r_{0} N \ln N \mid X_{N}=\frac{r_{0}+r_{1}}{2} N \ln N\right) \cdot P\left(X_{N}>\frac{r_{0}+r_{1}}{2} N \ln N\right) \text {. (2) }
$$

When $X_{N}=\frac{r_{0}+r_{1}}{2} N \ln N, Z_{N}$ follows binonial distribution with Bernoulli trials $\frac{r_{0}+r_{1}}{2} N \ln N$, and success probability $P\left(Y_{N}>p_{0} N^{\alpha k}\right)$. UseGngtation $W_{N}$ to stand for this distribution be $W_{N}$, its expected value is $\frac{r}{2} N \ln N \cdot P\left(Y_{N}>p_{0} N^{\alpha k}\right)$, so

$$
\begin{aligned}
& P\left(Z_{N}>r_{0} N \ln N \mid X_{N}=\frac{r_{0}+r_{1}}{2} N \ln N\right) \\
& >1-P\left(\left|W_{N}-\frac{r_{0}+r_{1}}{2} N \ln Q P\left(Y_{N}>p_{0} N\right)\right| \geq\left(\frac{r_{0}+r_{1}}{2} N \ln N \cdot P\left(Y_{N}>p_{0} N^{\alpha k}\right)-r_{0} N \ln N\right)\right) \\
& \geq 1-\frac{\frac{r_{0}+r_{1}}{2} N \ln N \cdot P\left(Y_{N}>p_{0} N^{\alpha k}\right) \cdot\left(1-P\left(Y_{N}>p_{0} N^{\alpha k}\right)\right)}{\left.\left(\frac{6+r_{1}}{2} N \ln N P\left(Y_{N}>p_{0} N^{\alpha k}\right)-r_{0} N \ln N\right)\right)^{2}} \text {. }
\end{aligned}
$$

With $N$ growing, the above formula goes to 1 , because $P\left(X_{N}>\frac{r_{0}+r_{1}}{2} N \ln N\right)$ in formula (2) also goes to 1, we get formula (1) goes to 1 . We verified condition 1.

To verify condition 2 :

Let elements (instances) in set $\mathrm{A}$ be $a_{1}, \ldots, a_{s}$, where $s$ is the number of elements (instances). Actually each instance of model RA has a probability, which is the probability it shows up when we generate an instance. Limited to A, each instance in A has a conditional probability, denote the conditional probability by $\mu\left(a_{i}\right), i=1, \ldots, s$, we have $\sum_{i=1}^{s} \mu\left(a_{i}\right)=1$. For instances of model RB*, constraints have an order, because constraints are selected one by one. Here we ignore the order, instances which have the same constraints are looked as one instance. Now denote the instances by $b_{1}, \ldots, b_{l}$, where $l$ is the number of instances. Easy to know every instance in model $\mathrm{RB}^{*}$ has probability $\mu\left(b_{j}\right)=\frac{1}{l}, j=1, \ldots, l$. We say that 
instance $a$ is stronger than instance $b$, if $a$ can be gotten from $b$ by putting some constraints and incompatible joint values in. Instances of model RB* contain $r_{0} N \ln N$ constraints, each constraint contains $p_{0} N^{\alpha k}$ incompatible joint values, so every instance $a_{i}$ in set A is stronger than some instances of model RB*. Let matrix $c$ be of size $s \times l$, let $c_{i j}=0$ if $a_{i}$ is not stronger than $b_{j}, c_{i j}=1$ if $a_{i}$ is stronger than $b_{j}$. When $a_{i}$ is stronger than $b_{j}$,

$$
P\left(a_{i} \text { is satisfiable }\right) \leq P\left(b_{j} \text { is satisfiable }\right) .
$$

So

$$
P\left(a_{i} \text { is satisfiable }\right) \leq \frac{1}{\sum_{h=1}^{l} c_{i h}} \sum_{j=1}^{l} c_{i j} P\left(b_{j} \text { is satisfiable }\right) .
$$

So

$$
\begin{aligned}
& P(\text { instances in } A \text { is satisfiable })=\sum_{i=1}^{s} \mu\left(a_{i}\right) P\left(a_{i} \text { is satisfiable }\right) \\
& \leq \sum_{i=1}^{s} \mu\left(a_{i}\right) \frac{1}{\sum_{h=1}^{l} c_{i h}} \sum_{j=1}^{l} c_{i j} P\left(b_{j} \text { is satisfiable }\right) \\
& =\sum_{j=1}^{l} \sum_{i=1}^{s} \mu\left(a_{i}\right) \frac{1}{\sum^{l} c_{i h}} P\left(b_{j} \text { is satisfiable }\right) .
\end{aligned}
$$

Symmetry will simplify the above formula. By "symmetry" we mean $\sum_{i=1}^{s} \mu\left(a_{i}\right) \frac{1}{\sum_{h=1}^{l} c_{i h}} c_{i j}$ are all equal for any $j, j=1, \cdots, l$. What we actually do in (3) is to split $P\left(a_{i}\right.$ is satisfiable) into $\sum_{h=1}^{l} c_{i h}$ pieces each piece compare with a $\frac{1}{\sum_{h=1}^{l} c_{i h}} P\left(b_{j}\right.$ is satisfiable $)$ where $c_{i j}=1$, then

$$
\mu\left(a_{i}\right) P\left(a_{i} \text { is satisfiable }\right) \leq \mu\left(a_{i}\right) \frac{1}{\sum_{h=1}^{l} c_{i h}} \sum_{j=1}^{l} c_{i j} P\left(b_{j} \text { is satisfiable }\right) .
$$

"Symmetry" ays that every $P\left(b_{j}\right.$ is satisfiable $)$ corresponds to $\sum_{i=1}^{s} \mu\left(a_{i}\right) \frac{1}{\sum_{h=1}^{l} c_{i h}} c_{i j}$ equally.

This is because no $b_{j}$ have reasons to be different, we will show "symmetry" in detail.

We only need to prove for $m, n \in\{1, \ldots, l\}, \sum_{i=1}^{s} \mu\left(a_{i}\right) \frac{1}{\sum_{h=1}^{l} c_{i h}} c_{i m}=\sum_{i=1}^{s} \mu\left(a_{i}\right) \frac{1}{\sum_{h=1}^{l} c_{i h}} c_{i n}$.

Let $b_{m}, b_{n}$ contain constraints $C_{m, 1}, \ldots, C_{m, r_{0} N \ln N}$ and $C_{n, 1}, \ldots, C_{n, r_{0} N \ln N}$. Constraints $C_{m, i}$, $i=1, \cdots, r_{0} N \ln N$, have incompatible joint values $m_{m, i, 1}, \ldots, m_{m, i, p_{0} N^{\alpha k}}$, similarly $C_{n, i}$ have 
incompatible joint values $m_{n, i, 1}, \ldots, m_{n, i, p_{0} N^{\alpha k}}$. Based on $b_{m}, b_{n}$, we define a bijection from instances to instances. Firstly, based on $b_{m}, b_{n}$, we define:

(1) A bijection from feasible constraints to feasible constraints, satisfied that $C_{m, 1}, \ldots, C_{m, r_{0} N \ln N}$ are paired with $C_{n, 1}, \ldots, C_{n, r_{0} N \ln N}$ respectively.

(2) For each constraint pair $C_{m, i} \mapsto C_{n, i}, i=1, \ldots, r_{0} N \ln N$, define a bijection from feasible joint values to feasible joint values, satisfied that $m_{m, i, 1}, \ldots, m_{m, i, p_{0} N^{\alpha k}}$ are paired with $m_{n, i, 1}, \ldots, \quad m_{n, i, p_{0} N^{\alpha k}}$ respectively.

Then, in this way, we defined a bijection from instances to instances, and we knothat is paired with $b_{n}$.

For every $a_{i}$, which is stronger than $b_{m}$ (or to say $c_{i m}=1$, there is a $a$, which is paired with $a_{i}$ in above bijection. We get that $a_{j}$ also is stronger than $b_{n}$ (or to say $c_{j m}=1$ ), and easy to know $\mu\left(a_{i}\right)=\mu\left(a_{j}\right)$. Furthermore when $a_{i}$ s paired with $a_{j}$, easy to know $\sum_{h=1}^{l} c_{i h}=\sum_{h=1}^{l} c_{j h}$. So $\mu\left(a_{i}\right) \frac{1}{\sum_{h=1}^{l} c_{i h}} c_{i m}=\mu\left(a_{j}\right) \frac{1}{\sum_{h=1}^{l} c_{j h}} c_{j m}$. Considering about the bijection, we find

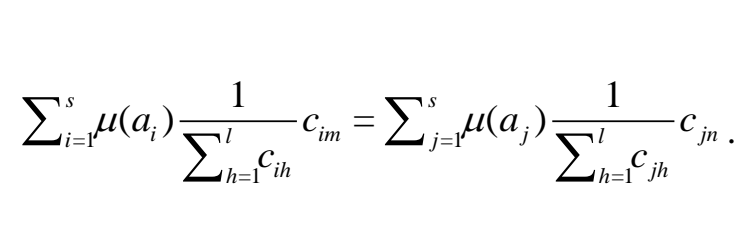

"Symmetry" holds.

And because

$$
\sum \sum_{j=1} \mu\left(a_{i}\right) \frac{1}{2 \sum_{h=1}^{l} c_{i h}} c_{i j}=\sum_{i=1}^{s} \mu\left(a_{i}\right) \frac{1}{\sum_{j=1}^{l} c_{i j}} \sum_{j=1}^{l} c_{i j}=\sum_{i=1}^{s} \mu\left(a_{i}\right)=1,
$$

so for any $j, \sum_{i=1}^{s} \mu \frac{1}{\sum_{h=1}^{l} c_{i h}} c_{i j}=\frac{1}{l}$. So by (3)

$$
\begin{aligned}
& P(\text { instances in A is satisfiable }) \leq \sum_{r=1}^{l} \frac{1}{l} P\left(b_{r} \text { is satisfiable }\right) \\
& =\sum_{r=1}^{l} \mu\left(b_{r}\right) P\left(b_{r} \text { is satisfiable }\right)=P(\text { instances in } R B * \text { is satisfiable }) \text {. }
\end{aligned}
$$

We verified condition 2 .

Lemma 4. Instances of model RA with $\alpha>0,0<p<1, r<\frac{\alpha}{-\ln (1-p)}, k \geq 3$ w.h.p. ${ }^{1}$ are satisfiable.

\footnotetext{
1 'with high probability'(w.h.p.) means that the probability of some event tends to 1 , as $N \rightarrow \infty$.
} 
Proof: $\frac{\alpha}{-\ln (1-p)}$ is a continuous and decreasing function with argument $p$, so there is $p_{1}$, satisfied $p<p_{1}<1$ and $r<\frac{\alpha}{-\ln \left(1-p_{1}\right)}<\frac{\alpha}{-\ln (1-p)}$. Let $r_{1}$ satisfy $r<r_{1}<\frac{\alpha}{-\ln \left(1-p_{1}\right)}$, then we have $p<p_{1}, r<r_{1}$, and $r_{1}<\frac{\alpha}{-\ln \left(1-p_{1}\right)}$. According to lemma 1 , instances of $\mathrm{RB}\left(r_{1}, p_{1}\right)$ w.h.p. is satisfiable. By lemma 2, easy to know instances of $\mathrm{RB}^{*}\left(r_{1}, p_{1}\right)$ w.h.p. is satisfiable. Finally by lemma 3, instances of $\mathrm{RA}(r, p)$ w.h.p. is satisfiable.

Theorem 1. Model RA has the same satisfiability transition as model RB. Specifically, let $r_{c r}=-\frac{\alpha}{\ln (1-p)}$ and parameters satisfied $\alpha>\frac{1}{k}, 0<p<1, k \geq \frac{1}{1-p}$, and $X$ is the number of solutions of an RA instance, then

$$
\begin{aligned}
& \lim _{n \rightarrow \infty} \operatorname{Pr}(X>0)=1 \text { when } r<r_{c r} \\
& \lim _{n \rightarrow \infty} \operatorname{Pr}(X>0)=0 \text { when }>r .
\end{aligned}
$$

Proof; We can get formula (4) by lemma 4. With the same method, we can get a similar lemma, and get formula (5).

\section{Experiments on model RA and RB}

Back-free algorithm and randôm walk algorithm are fundamental algorithms for CSPs. A lot of theoretical works have been done about them. [22] proved back-free algorithm with unit clause heuristic can solve 3-SAT with positive probability, when constraint density below 2.9. [23] proved back-free algonthm with pure literal heuristic can w.h.p. solve 3-SAT, when constraint density below 1.63. [24] proved random walk algorithm with pure literal heuristic can w.h.p. solve 3-SAT, when constraing density below 1.63.

We will give steps of those two algorithms, one is random walk algorithm, the other is a kind of back-free algorithm. By applying them to model RB and RA, the results show that when $N$ is not very small, model RA and model RB are almost the same.

Algorithm 1 (random yalk):

1. Give a random assignment. Set a maximum number of steps.

2. If current assignment satisfies all constraints, terminate the algorithm, output current assignment. Else randomly select a constraint, and a variable in the constraint, reassign it a value.

3. Repeat step 2, until repeat time has gotten to the maximum number of steps, output 'fail'.

Every dot in Figure 2 and Figure 3 is an average of 10 trials, the maximum number of steps is 2000 . We see that when $r$ is small, problems can be solved by algorithm 1 with probability 1. Then the probabilities fall to 0 dramatically. The fallings happen almost at the same $r$ values on model RB and RA. 


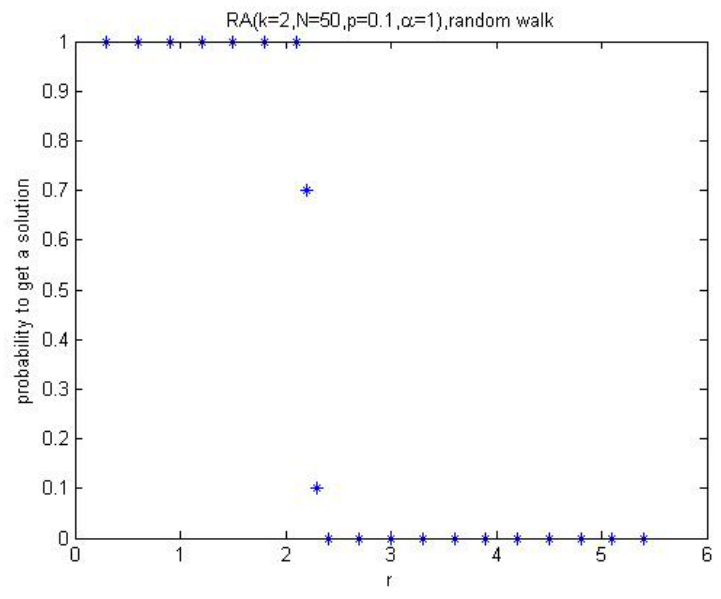

Figure 2. Algorithm 1 on model RB

Algorithm 2 (back-free).

1. Assign $\sigma_{1}$ a random value.

To each $\sigma_{2}, \sigma_{\mathrm{N}}$ successively do $2 、 3 、 4$

2. Assign the current variable a random value.

3. If current variable is well assigned (do not violate any constraints with already assigned variables), output the solution, terminate the algorithm, else record the value and reassign it an unrecorded value.

4. If all values in domain are recorded, output "fail", terminate the algorithm, else repeat 3.

Every dot in Figure 4 is an average of 15 trials, Every dot in Figure 5 is an average by 20 trials. As we can see, probabilities to get a solution have similar shapes on model RB, and on model RA. This experiment also shows RA and RB are very similar. 
International Journal of Hybrid Information Technology

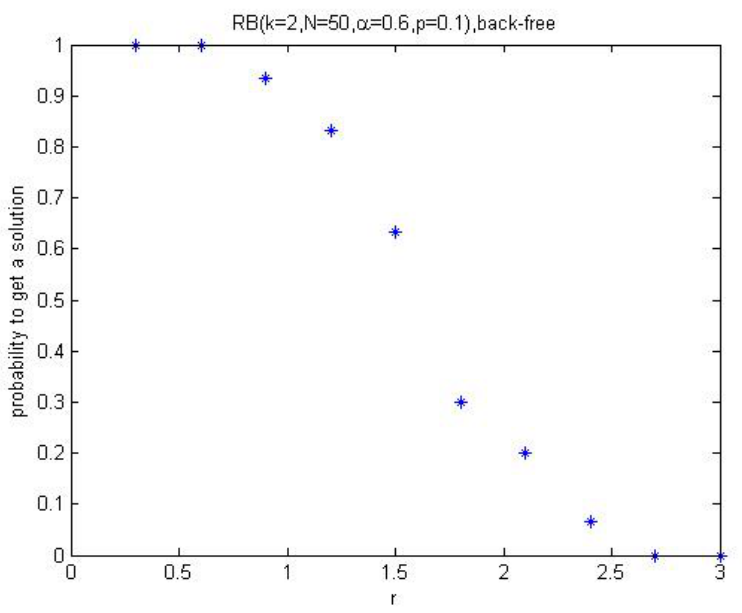

Figure 4. Algorithm 2 on model RB

Actually in an unpublished manuscript, we proved that the probability to get a solution of algorithm 2 on model RA is:

\section{Figure 5. Algorithm 2 on model RA}

$$
\prod_{i=k}^{N}\left(\sum_{j=0}^{c_{i-1}^{k-1}}\left(1-\left(1-(1-p)^{j}\right)^{N^{\alpha}}\right) \operatorname{bin}\left(j, C_{i-1}^{k-1}, \frac{r N \ln N}{C_{N}^{k}}\right)\right)
$$

where $\operatorname{bin}(j, x, p)=C_{x}^{j} p^{j}(1-p)^{x-j}$. And when $r+\frac{2 / 3+\sqrt{4 / 9+8 r k}}{2 k}<\frac{\frac{1}{k} \alpha}{-\ln (1-p)}$, this probability goes to 1 , as $N$ growing. 


\section{Conclusion}

Since revised models were proposed, studies are mainly on model RB, RD. In this paper we proved that model RA has the same satisfiability transition as model $\mathrm{RB}$, when $k \geq 3$. The similar conclusion can also be extended to model RC. Experiments on model RA and RB show that those two models are very similar. For particular questions, model RA may be easier to analyze, so this paper provides basic properties of model RA.

Studies on CSP models shed light on computer science and information technology. More works should be done, such as satisfiability transition study of model RA when $k=2$, such as algorithm analyses on those models. And we think the future work would be not only on a limitation situation, but also detailedly how properties change along lines of variable-domain plane in Figure 1.

\section{References}

[1] F. Rossi, P. Van Beek and T. Walsh, "Handbook of Constraint"Programnting", New York: Elsevier Science Inc., (2006).

[2] B. M. Smith and M. E. Dyer, "Locating the Phase Transition in Binary Constraint Satisfaction Problems", Artif Intell, vol. 81, (1996), pp. 155-181.

[3] I. Gent, E. Macintype, P. Prosser, B. Smith and T. Walsh, "Random constraint satisfaction: flaws and structure", Constraints, vol. 6, no. 4, (2001), P. 345-372.

[4] D. Achlioptas, L. Kirousis, E. Kranakis, D Krizanc, M. Molloy and Y. Stamatiou, "Random constraint satisfaction: a more accurate picture”. Proceedings of CP'97, (1997), p`p. 107-120.

[5] K. Xu and W. Li, "Exact Phase Transitions in Randon Constraint Satisfaction Problems", Journal of Artificial Intelligence Research, vol 12,-(2000), bp. 93-103.

[6] B. Smith, "Constructing an asymptotic phase transition in random binary constraint satisfaction problems", Theoretical Computer Science, vøl. 265. (2001), pp. 265-283.

[7] A. Frieze and M. Molloy, "The satisfiability threshold for randomly generated binary constraint satisfaction problems", Proceedings of Randon '03, (2003), pp. 275-289.

[8] Y. Gao and J. Culberson, “Consistency and random constraint satisfaction models with a high constraint tightness' 'Proceedings of CP '04, (2004), pp. 17-31.

[9] P. Cheeseman, B Kanefsky and W. Taylor, "Where the really hard problems are", In Proceedings of IJCAI-91, (1991), pp. 331-337.

[10] E. Friedgut and an appendix Dy J. Bourgain, "Sharp Thresholds of Graph Properties, and the k-sat Problem", JAmer. Math. Soc., vol. 12, no. 4, (1999), pp. 1017-1054.

[11] D. Achlioptas and F. Ricel-Tersenghi, "On the Solution-Space Geometry of Random Constrain Satisfaction Problems', in Proceedings of STOC'06, (2006), pp. 130-139.

[12] M. Mézard, G. Parisi and R. Zecchina, "Analytic and Algorithmic Solutions of Random Satisfiability Problems", Science, vol. 297, (2002), pp. 812.

[13] A. Montanari, F. Ricci-Tersenghi and G. Semerjian, "Clusters of Solutions and Replica Symmetry Breaking in Random k-satisfiability", J. Stat. Mech.: Theor. Exp., (2008), P04004.

[14] K. Xu and W. Li, "Many Hard Examples in Exact Phase Transitions", Theoretical Computer Science, vol. 355, (2006), pp. 291-302.

[157 K. Xu, F. Boussemart, F. Hemery and C. Lecoutre, "Random Constraint Satisfaction: Easy Ceneration of Hard (Satisfiable) Instances", Artificial Intelligence, vol. 171, (2007), pp. 514-534.

[16] C. Lecoutre, "Constraint Networks: Techniques and Algorithms", London: ISTE Ltd, Hoboken: John Wiley \& Sons Inc., (2009).

[17] C. Y. Zhao, P. Zhang, Z. M. Zheng and K. Xu, "Analytical and Belief-propagation Studies of Random Constraint Satisfaction Problems with Growing Domains", Phys Rev E, vol. 85, (2012), pp. 016106

[18] T. Liu, X. Lin, C. Wang, K. Su and K. Xu, "Large Hinge Width on Sparse Random Hypergraphs", IJCAI 2011: 611-616, AAAI, ( 2011). 
[19] C. Y. Zhao and Z. M. Zheng, "Threshold behaviors of a random constraint satisfaction problem with exact phase transitions", Information Processing Letters, vol. 111, (2011), pp. 985-988.

[20] S. Richter, M. Helmert and C. Gretton, "A stochastic local search approach to vertex cover", In: Proceedings of the 30th German Conference on Artificial Intelligence (KI), (2007).

[21] E. Alphonse and A. Osmani, "A model to study phase transition and plateaus in relational learning", The 18th International Conference on Inductive Logic Programming, (2008), pp. 6-23.

[22] M. T. Chao and J. Franco, "Probabilistic Analysis of Two Heuristics for the 3-satisfiability Problem”, SIAM J. Comput., vol. 15, no. 4, (1986), pp. 1106-1118.

[23] A. Z. Broder, A. M. Frieze and E. Upfal, "On the Satisfiability and Maximum Satisfiability of Random 3-CNF Formulas", in: 4th Annual ACM-SIAM Symp. on Discrete Algorithms, Austin, TX, (1993), ACM, New York, pp. 322-330.

[24] M. Alekhnovich and E. Ben-Sasson, "Linear Upper Bounds for Random Walk on Small Density Random 3-cnfs”, SIAM J Comput, vol. 36, no. 5, (2006), pp. 1248-1263.

Author

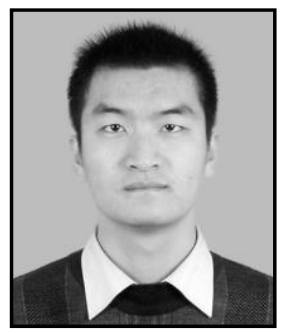

\section{Wei Xu}

He received his M.Sc. in Mathematics (2009) from University of Science and Technology Beijing. Now he is a PhD student at School of Automation and Electrical Engineering. University of Science and Technology Beijing. His current research interests include different aspects of Theory of Computation.

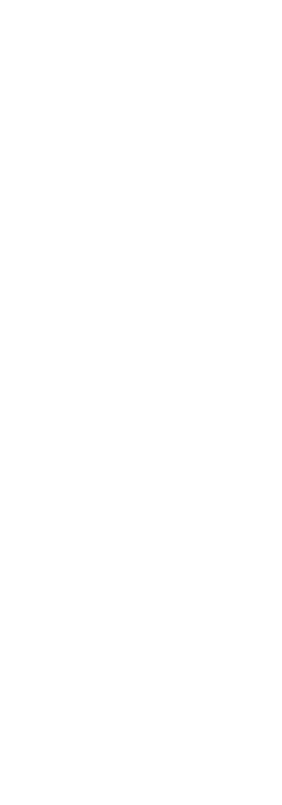

